International Journal of Applied Mathematics

Volume 26 No. $3 \quad 2013,379-390$

ISSN: $1311-1728$ (printed version); ISSN: 1314-8060 (on-line version)

doi: http://dx.doi.org/10.12732/ijam.v26i3.11

\title{
FINITE ELEMENT METHODS BASED ON GENERALIZED \\ EXPO-RATIONAL B-SPLINES WITH HARMONIC POLYNOMIAL COEFFICIENTS
}

\author{
Peter Zanaty \\ Faculty of Technology \\ Narvik University College \\ P.O. Box 385, 8505 Narvik, NORWAY
}

\begin{abstract}
We present an upgrade of a recent work [7] where the performance of several novel Partition of Unity Methods (PUM) [2] were examined on non-degenerate triangulations for solving linear elliptic partial differential equations. The current study focuses on changing the local polynomials as functional coefficients in the Partition of Unity Methods (PUM) from Taylor expanding polynomials to harmonic polynomials. We provide numerical results to illustrate the behavior of the PUM when switching from local Taylor polynomials to local harmonic polynomials. These results support the conclusion that on sufficiently refined meshes harmonic polynomials tend to outperform Taylor polynomials.
\end{abstract}

AMS Subject Classification: 65 N30, 65N38, 31A05

Key Words: harmonic polynomials, finite element, partition of unity method, expo-rational B-spline, Galerkin-Bubnov method

\section{Introduction}

Partition of unity methods (PUM) were introduced in finite-element analysis by Babuška and Melenk in [2]. The motivation behind this technique is to be able 
to tailor the ansatz spaces to suit the specifics of the concrete problem in consideration. In the present paper we shall consider several recently introduced PUM and will compare their numerical performance on a model boundary-value problem for Laplace's equation. In this study we duplicate the problem setting from the study [7], the chosen boundary-value problem is of mixed type: the boundary data are of Dirichlet type on part of the boundary and of Neumann type on the remaining part of the boundary. We will restrain our investigation to Galerkin-Bubnov projection methods of approximation. The study is complemented with the consideration of a second benchmark problem, where Dirichlet boundary-value data are given on the boundary of a non-rectangular domain.

The choice of harmonic polynomials as local (or enrichment) functions for the Laplace's equation arises from the fact that this space of polynomials is the kernel of Laplacian operator on the polynomials, hence compared to Taylor expanding polynomial spaces of the same dimension their local approximation power is superior. This property, combined with the property of the PUM methods allows for a possible upgrade of the methods described in [7].

Our investigation is focusing on the change of local functions in the Bubnov version of the Galerkin method where the projection space and the test space are spanned by the same basis. In this case the inner product in the entries of the stiffness matrix for both the Galerkin and least-squares FEM involve derivatives of the same functions, which provides common ground for comparison between the results of the two types of FEM.

\section{Preliminaries}

In this section we shall briefly describe the two partitions of unity utilized by our investigation and give an outline of the applied harmonic polynomials. Both methods build on the theory of Expo-rational B-splines (ERBS) introduced in [4], which provides basis functions capable of arbitrary degree Hermite interpolation at the interpolation nodes. Usually these spaces are enriched with the use of local Taylor expanding polynomials resulting in Hermite interpolating curves

$$
C(t)=\sum_{i} \ell_{i}(t) \Gamma_{i}(t),
$$

where $\Gamma_{i}(t)$ is the ERBS basis function and $\ell_{i}(t)$ is the corresponding local (enrichment) function.

The first method described applies a very general $C^{\infty}$-smooth construction 
building on ERBS, while the second version is a triangular polynomial partition of unity allowing Hermite interpolation at the vertices, but the triangular elements meet only with $C^{0}$-continuity over the edges.

\subsection{Smooth Expo-Rational Partition of Unity}

The smooth ERBS PUM applied in our study is a special case of a tensorproduct variant of the $C^{\infty}$-smooth constructions presented in [3].

This partition of unity is constructed on a scattered point set $\left(\left\{v_{i}=\right.\right.$ $\left.\left.\left(x_{i}, y_{i}\right), i=1, \ldots, N\right\}\right)$ using two sets of radii for each point $0<\rho_{i}<R_{i}$. Utilizing the Expo-rational basis function centered at zero and supported at $[-1,1](B(0)=1, B(1)=0, B(-1)=0)$ the method invokes two sets of auxiliary functions $\varphi_{i}(x, y)=B\left(\frac{x-x_{i}}{\rho_{i}}\right) B\left(\frac{y-y_{i}}{\rho_{i}}\right)$ and $\psi_{i}(x, y)=B\left(\frac{x-x_{i}}{R_{i}}\right) B\left(\frac{y-y_{i}}{R_{i}}\right)$. The requirement for these auxiliary functions (i.e. for the sets of radii) is that the support of $\psi$ functions has to cover $\Omega$, while each point $v_{i}$ should only be inside the support of its respective $\varphi$ function, $\varphi_{i}$.

In this study we restrict the positive parameters $\varrho_{i}, i=1, \ldots, N$, so that supp $\varphi_{i} \cap \operatorname{supp} \varphi_{j}=\emptyset, i \neq j$, i.e., making the smaller radii circles non overlapping, yielding the following basis functions [3]

$$
\Gamma_{i}(u)=\varphi_{i}(u)+\frac{\psi_{i}(u)}{\sum_{k=1}^{N} \psi_{k}(u)}\left(1-\sum_{j=1}^{N} \varphi_{j}(u)\right),
$$

$i=1, \ldots, N, u \in \Omega$.

\subsection{Triangular Beta Function B-Spline Partition of Unity}

The Beta function B-spline (BFBS) based partition of unity technique, described in [5], provides a family of polynomial triangular basis functions. The construction allows Hermite interpolation at the vertices of arbitrary multiplicity, but the triangular elements meet only with $C^{0}$-continuity over the edges.

The bases are constructed triangle-by-triangle, integrating the incomplete Beta function corresponding to the desired Hermite multiplicities as a weight function over a subdivided simplex. Let us denote $\sum_{3}\left(v_{1}, v_{2}, v_{3}\right)$ to be a triangle with vertices $v_{1}, v_{2}, v_{3}$. Given a triangle $\Omega=\sum_{2}\left(v_{1}, v_{2}, v_{3}\right)$ and a point $u$ the $i^{\text {th }}$ base with multiplicity $\mu$ is defined as:

$$
\Gamma_{i}^{\mu}(u)=\frac{\int_{\Omega_{i}(u)} B_{\mu}^{\Omega}(v) \mathrm{d} v}{\int_{\Omega} B_{\mu}^{\Omega}(v) \mathrm{d} v},
$$


where $\Omega_{i}(u)$ is the $i^{\text {th }}$ sub-triangle of $\Omega$ using $u$ as a new vertex replacing $v_{i}$ (i.e., $\left.\Omega_{2}(u)=\sum_{3}\left(v_{1}, u, v_{3}\right)\right)$ and $B_{\mu}^{\Omega}(v)$ is the Bernstein polynomial with the multiindex $\mu=\left(m_{1}, m_{2}, m_{3}\right)$ on triangle $\Omega$, defined by

$$
B_{\mu}^{\Omega}(v)=\frac{|\mu| !}{\mu_{1} ! \mu_{2} ! \mu_{3} !} b_{1}^{\mu_{1}} b_{2}^{\mu_{2}} b_{3}^{\mu_{3}},
$$

where $b$ denotes the barycentric coordinates of $v$ in the triangle $\Omega, v=b_{1} v_{1}+$ $b_{2} v_{2}+\left(1-b_{1}-b_{2}\right) v_{3}$.

After carrying out the integration we arrive to the polynomial basis functions

$$
\Gamma_{i}^{\mu}(v)=b_{i} \sum_{\gamma=\delta_{i} \mu}^{\mu} B_{\gamma}^{\Omega}(v),
$$

where the summation goes through a multindex $\gamma: 0 \leq \gamma_{j} \leq \mu_{j}, j \neq i$ and $\gamma_{i}=\mu_{i}$, and where $b_{i}$ denotes the $i$-th barycentric coordinate of $v$ in triangle $\Omega$.

\subsection{Harmonic Polynomials}

Harmonic polynomials of degree $d$ are bivariate polynomials, whose Laplacian is zero

$$
\mathcal{H}_{d}=\left\{p: \triangle p=0, p \in \mathcal{P}_{d}\right\} .
$$

For each degree $d, \mathcal{H}_{d}$ consists of two types of basis for harmonic polynomials, which can be written in polar coordinates as $r^{k} \cos (k \theta)$ and $r^{k} \sin (k \theta)$, $k=0, \ldots, d$ (if $k=0$ both types of bases degenerate to constant 1 ). Thus, the harmonic polynomials of degree $d$ have dimension of exactly $2 d+1$ in contrast to the whole family of polynomials of the same degree, which span $\frac{(d+1)(d+2)}{2}$ dimensions. This reduction of dimension combined with the same approximation properties makes this family of polynomials an attractive choice for local polynomials in the case where the function space to be approximated consists of harmonic functions.

Bases which provide Hermite interpolation of degree two conserve the Laplacian (or any other second order differential operator) of the local function at the vertex, as all the involved derivatives of the base function are zero at the vertex.

\section{Comparison of the Performance}

In this section we present two benchmark problems and provide numerical results of the performance of the generalized Expo-rational based FEM's when 


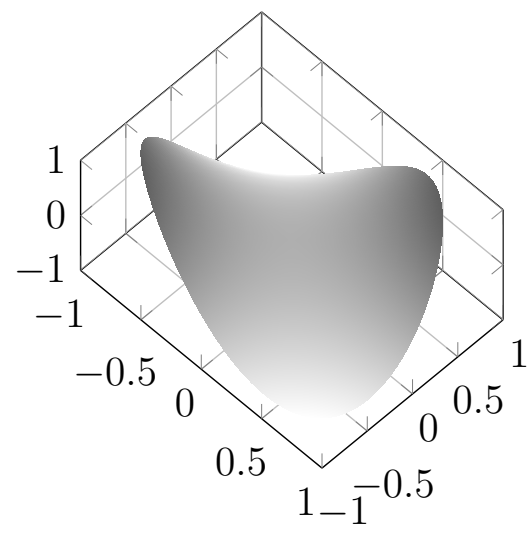

(a) $H_{2,2}$

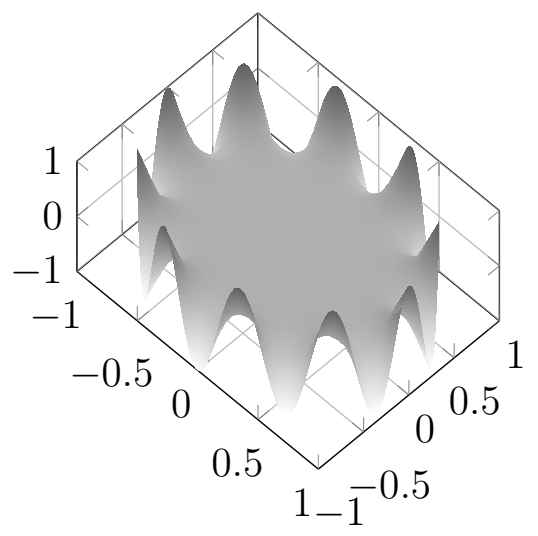

(b) $H_{10,1}$

Figure 1: Harmonic polynomials on the unit disk.

using local Taylor and harmonic polynomials.

\subsection{Problem Setting 1}

The first problem setting was introduced in [7]. The benchmark Laplace BVP is given on a square boundary by

$$
\begin{aligned}
& \mathcal{A}(\Phi) \equiv \triangle \Phi=0, \quad \text { in } \Omega=[0,1]^{2} \\
& \mathcal{B}(\Phi) \equiv\left\{\begin{array}{ll}
\Phi=0 & \text { on } \Gamma_{\Phi}=\{(x, y): x \in\{0,1\}, y \in[0,1]\} \\
\frac{\partial \Phi}{\partial n}+q=0 & \text { on } \Gamma_{q}=\{(x, y): x \in(0,1), y \in\{0,1\}\}
\end{array},\right.
\end{aligned}
$$

where $q=-\left(\left(1+e^{-\pi}\right) y-e^{-\pi}\right) \pi \sin \pi x$. The unique analytic solution for this equation is

$$
\Phi(x, y)=e^{(y-1) \pi} \sin x \pi .
$$

The considered example is a Poisson equation of Laplacian type with homogeneous Dirichlet boundary-value data $\left(Q=0\right.$ and $\bar{\Phi}=0$ on $\left.\Gamma_{\phi}\right)$. This selection of the model BVP has allowed for all types of bases in [7] to meet the essential boundary conditions by simply forcing the respective coefficients to zero. Generally, meeting the Dirichlet boundary value by forcing the local coefficients is not this straightforward, but in this special tensor-product case the use of local harmonic polynomials will still allow a similar substitution without the complete loss of approximation around the boundary. The required interpolation of the function on the respective segment of the boundary for the chosen Dirichlet 


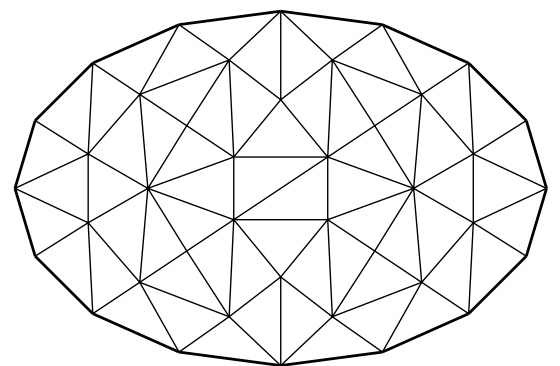

(a) Step 4

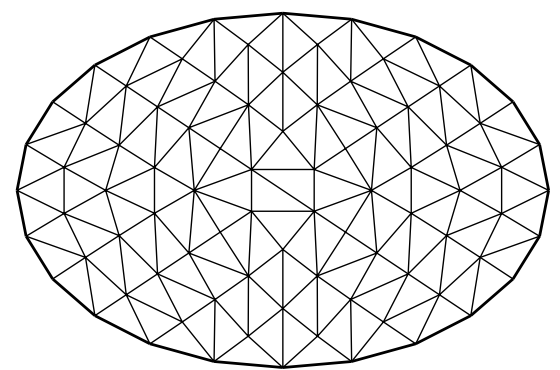

(b) Step 6

Figure 2: Tessellation of the ellipse. This figure shows two examples of meshes resulting from iterated tessellation of the domain in Problem setting 2.

boundary-value data can therefore still be done exactly by setting respective coefficients to zero.

\subsection{Problem Setting 2}

The domain of the first benchmark BVP is very simple and by nature it favors tensor-product approximations. Also, the axis of the local polynomial space and the Dirichlet boundary segments coincide, which allows for a very simple and effective local polynomial approximation there. Furthermore the Dirichlet boundary-value data is homogeneous, which simplifies the handling of essential boundary conditions.

To extend the scope of the study, a new problem setting is considered for the triangular BFBS FEM's where the domain is a tessellation of an ellipse, and the boundary conditions are entirely of Dirichlet type with non-homogeneous data. The result of two steps of the tessellation process used in this problem setting can be seen in Figure 2 .

The domain of the second BVP is given by $\Omega=\left\{(x, y): \frac{x^{2}}{1.5^{2}}+y^{2} \leq 1\right\}$ while the unique analytic solution of the BVP is

$$
\Phi(x, y)=\log \left(x^{2}+(y-1.5)^{2}\right) .
$$




\subsection{Numerical Results}

The Galerkin-Bubnov FEM yields the following equation in both cases

$$
\sum_{a \in \mathcal{F}}\left(\int_{\Omega} \frac{d}{d x} N_{b} \frac{d}{d x} N_{a}+\frac{d}{d y} N_{b} \frac{d}{d y} N_{a} \mathrm{~d} \Omega\right) \tilde{\Phi}_{a}+\int_{\Gamma_{q}} N_{b} \bar{q} \mathrm{~d} \Gamma=0,
$$

for each base $b \in \mathcal{F}$. Note that for the second model problem the entire boundary condition is of Dirichlet type, therefore $\Gamma_{q}=\emptyset$.

To solve BVP (8) numerically for each of the two benchmark instances, we introduce a set of triangulations on $\Omega$ which is the same for all FEM considered in the respective problem setting (see Figure 2 for problem setting 2.). We compute the error as a function of the approximation step for each of the considered FEM. The error is measured in three different norms

1. $L_{2}$-norm: $\|f\|_{L_{2}(\Omega)}=\left(\int_{\Omega}|f(u)|^{2} \mathrm{~d} u\right)^{\frac{1}{2}}$.

2. Energy norm: $\|f\|_{e(\Omega)}=\left[\int_{\Omega}\left(|f(u)|^{2}+|\nabla f(u)|^{2}\right) \mathrm{d} u\right]^{\frac{1}{2}}$.

3. Sobolev-2 norm $\|f\|_{W_{2}^{k}(\Omega)}$ for $k=2$.

The triangular BFBS base provides only $C^{0}$ smoothness over the edges, therefore, the Sobolev-2 norm error measurements will only be displayed for the smooth ERBS construction.

The algorithm used for obtaining the results can be divided into 4 separate stages. The first stage is the numerical approximation of the stiffness matrix and load vectors in (11). As the PDE considered here has constant coefficients, the element stiffness matrices are only depending on the shape of the corresponding element. Furthermore, in the case of the polynomial bases, there is no need to use any cubatures as these integrals can be evaluated explicitly in a stable way with the use of Bernstein-Bézier basis. For the load vectors one has to evaluate integrals over the edges of the triangulation, in both cases the partition of unity basis functions simplify considerably there, which allows for application of a fast Gaussian quadrature rule designed specifically for the univariate basis functions as weight functions on these edges.

The second stage of the FEM is related to forcing the essential boundary conditions; this requires approximation of the boundary data with the local polynomials. This stage is straightforward for the first BVP, while for the second BVP the local functions are computed to be best approximating in L2 norm with a weight function of the corresponding partition of unity base. As there might be many different best approximations for a given boundary 


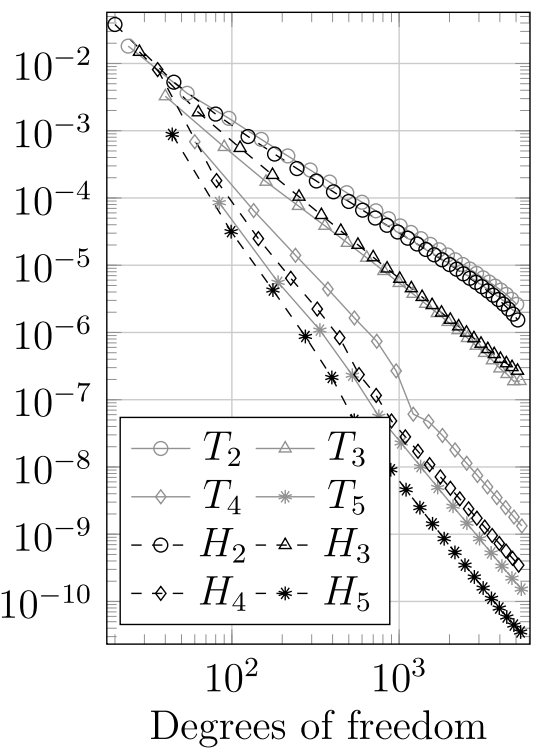

(a) $L^{2}$ norm

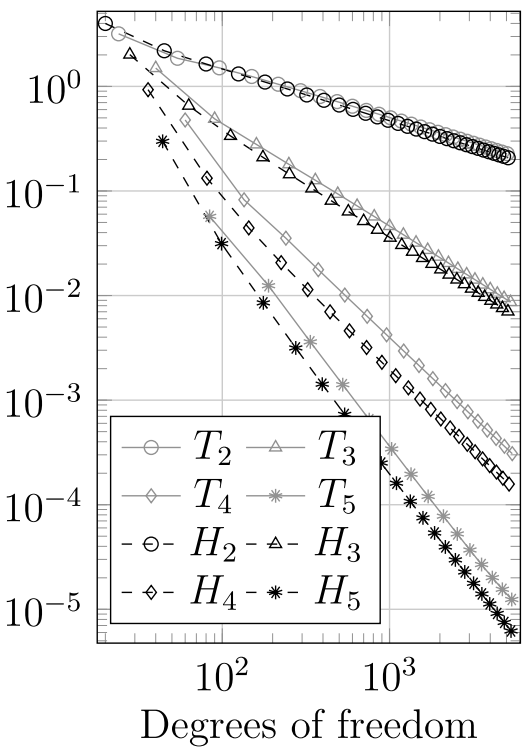

(b) $W_{2}^{2}$ norm

Figure 3: Error of ERBS PUM for problem 1. The solid gray lines $T_{2}, T_{3}, T_{4}, T_{5}$ show the performance of the smooth ERBS PUM with local Taylor polynomials, while the dashed lines $H_{2}, H_{3}, H_{4}, H_{5}$ show the performance of the same type of PUM with local Hermite polynomial enrichment.

function, it is favorable to avoid solutions with large coefficients, therefore, to improve stability an additional factor corresponding to the absolute sum of the coefficients is included in the minimization term.

The third stage is the solution of the FEM problem, i.e., calculating the coefficients by solving $K c=f$ linear equation from (11); the solution is obtained by the use of QR decomposition with column pivoting. The challenging part in the solution of this system of equations lies in the resulting large condition numbers of the considered PUM methods.

The last stage is the approximation of the error in the respective norms which is done by an adaptive Romberg numerical integration on all the elements. The Romberg method used is an extension of the univariate Romberg method, which applies hat functions and repeated Richardson extrapolation for multivariate integrals.

In Figure 3 are given the graphs of the dependence of the considered FEM 


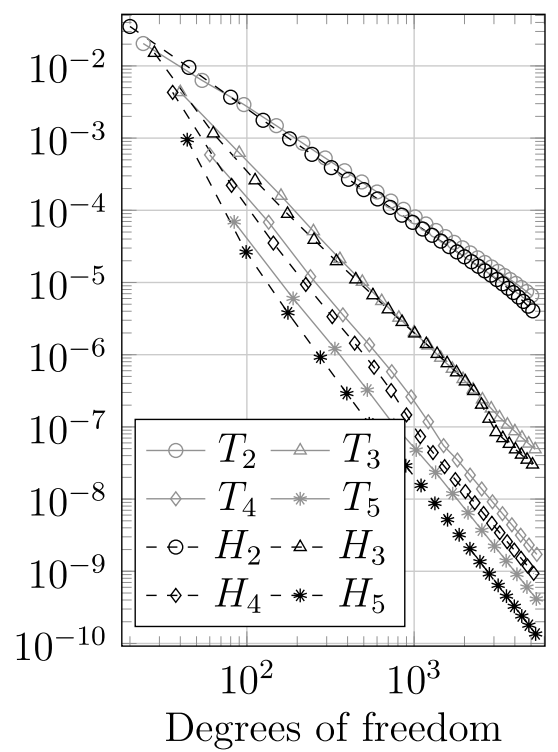

(a) $L^{2}$ norm

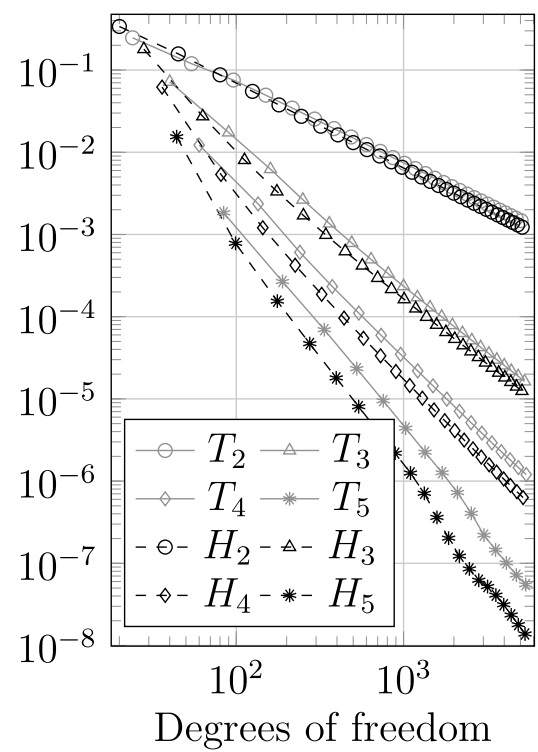

(b) Energy norm

Figure 4: Error of GERBS PUM for problem 1. The solid gray lines $T_{2}, T_{3}, T_{4}, T_{5}$ show the performance of the $C^{0}$ BFBS methods with local Taylor polynomials, while the dashed lines $H_{2}, H_{3}, H_{4}$, $H_{5}$ show the performance of methods with local Hermite polynomial enrichment.

when switching from local Taylor expanding polynomials to harmonic polynomials for the ERBS and the GERBS PUM, respectively for problem setting 1, while Figure 5 shows the similar plots for the GERBS PUM in problem setting 2. Each sequence represents a method with an indicated local polynomial degree on subsequent approximation steps, where the resolution of the considered uniform triangulation is increased. The $x$-axis of the error plots represents the total degree of freedom of the ansatz space of the FEM while the $y$-axis shows the error of the method in Energy norm.

\section{Concluding Remarks}

As the numerical results suggest, harmonic polynomials offer advantage over the Taylor polynomials in terms of error with similar degrees of freedom. This is due to the fact that when using ansatz spaces of similar dimension the harmonic 


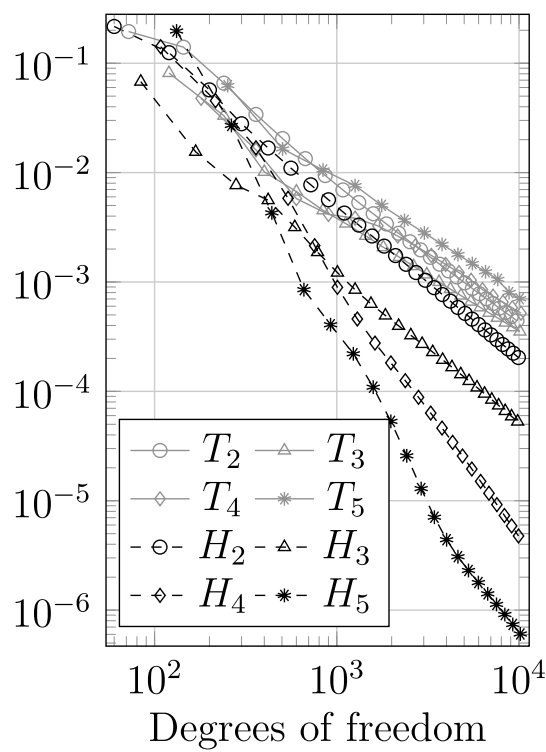

(a) $L^{2}$ norm

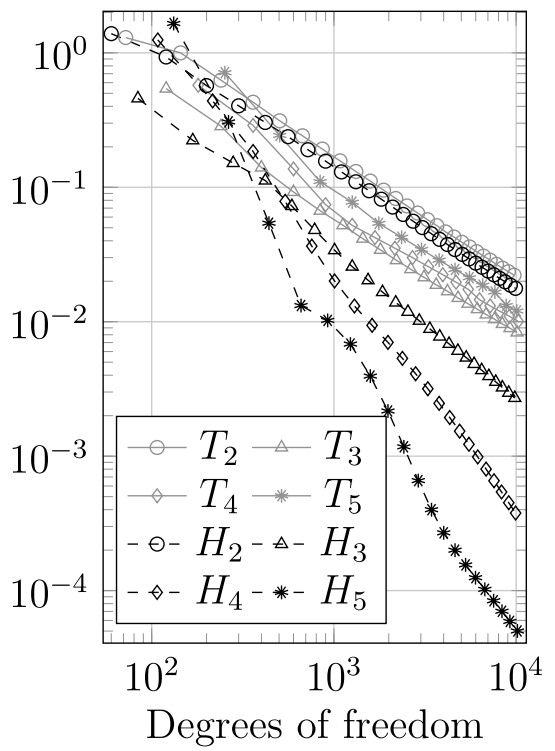

(b) Energy norm

Figure 5: Error of GERBS PUM for problem 2. The solid gray lines $T_{2}, T_{3}, T_{4}, T_{5}$ show the performance of the $C^{0}$ BFBS methods with local Taylor polynomials, while the dashed lines $\mathrm{H}_{2}, \mathrm{H}_{3}, \mathrm{H}_{4}$, $H_{5}$ show the performance of methods with local Hermite polynomial enrichment.

polynomials allow for a considerably higher degree of polynomial approximation. If the methods are compared for the same polynomial degree, then the Taylor polynomials will outperform the harmonic polynomials as the harmonic polynomials are a subspace of the Taylor polynomials and the partitions of unity used in these experiments do not conserve the Laplacian outside of the vertices of the mesh.

Figure 5 suggests that the increase of the complexity of the domain and the switch from a BVP on a cartesian-product domain to a general non-cartesianproduct domain tends to make the advantage of using local harmonic functions versus local Taylor polynomials more pronounced.

In [6] Melenk and Babuška studied the set of functions which satisfy a given differential equation as enrichment functions for the PUM in context of the same PDE, namely they conducted studies for the harmonic polynomials for Laplace's equation and introduced generalized harmonic polynomials for 
Helmholtz equation. The generalized harmonic polynomials are analogues of the harmonic polynomials for general elliptic equations with analytic coefficients.

Another interesting technique, the proper orthogonal decomposition (pod), was investigated in [1] for the purpose of generating enrichment functions for generalized FEM. The conclusion of the authors was that the use of the pod method yields local approximation spaces which provide significantly better results than the generic polynomial FEM expansions of similar degrees of freedom.

Some of the possible topics of future investigation are:

- Investigation of the use of generalized harmonic polynomials and proper orthogonal decomposition techniques with GERBS partitions of unity.

- Comparison of the performance of harmonic and Taylor polynomials in higher dimensions.

- Analysis of optimal partition of unity basis functions among GERBS basis functions.

- How would the use of combined refinement in the element size, $h$, and the polynomial degree, $d$ (hp-FEM) affect the performance gain of the local harmonic polynomials compared to Taylor polynomials?

- Investigation of the correspondence between the partition of unity and the resulting condition number of the stiffness matrix, with eventual addressing of preconditioning issues.

\section{References}

[1] W. Aquino, J.C. Brigham, CJ Earls, and N. Sukumar, Generalized finite element method using proper orthogonal decomposition, International Journal for Numerical Methods in Engineering, 79, No. 7 (2009), 887-906.

[2] Ivo Babuška, J.M. Melenk, The Partition of unity method, International Journal for Numerical Methods in Engineering, 40, No. 4 (1997), 727-758.

[3] Lubomir T. Dechevsky, Smooth convex resolution of unity and/or scattered-point Hermite-interpolation by generalized expo-rational Bsplines, I: General simply connected covers and partitions, Int. J. Pure Appl. Math., 65, No. 4 (2010), 391-412. 
[4] Lubomir T. Dechevsky, Arne Lakså, Børre Bang, Expo-rational B-splines, Int. J. Pure Appl. Math., 27, No. 3 (2006), 319-367.

[5] Lubomir T. Dechevsky, Peter Zanaty, Arne Lakså, Børre Bang, First instances of generalized expo-rational finite elements on triangulations, American Institute of Physics Conference Proceedings, 1410, No. 1 (2011), 49-61.

[6] J.M. Melenk, I. Babuška, Approximation with harmonic and generalized harmonic polynomials in the partition of unity method, Comput. Assist. Mech. Eng. Sci., 3, No. 4 (1997), 607-632.

[7] Peter Zanaty, Lubomir T. Dechevsky, On three finite element methods generated by Hermite-interpolating bases, Int. J. of Pure and Appl. Math., To Appear. 